\title{
A REMARK ON THE HARDY-LITTLEWOOD CONVERGENCE TEST FOR FOURIER SERIES
}

\author{
M. TOMIĆ
}

1. It is well known that the condition

$$
f\left(x_{0} \pm t\right)-f\left(x_{0}\right)=o\left(\frac{1}{|\log t|}\right) \quad(t \rightarrow+0)
$$

does not ensure the convergence of the Fourier series $\mathcal{S}[f]$ of $f(x)$ at the single point $x_{0}$. The Hardy-Littlewood convergence test [3, p. 63] gives the convergence of $\mathfrak{S}[f]$ at $x_{0}$ to the sum $f\left(x_{0}\right)$, provided that the condition (1) is satisfied, and the coefficients of $S[f]$ are $O\left(n^{-8}\right)$ for some $\delta>0$. By the use of Tauberian theorems for Valiron and Riesz method of summability, this test was generalized by HardyLittlewood, Morgan, Wang and Sunouchi [1].

The purpose of this note is to give a new proof of the Hardy-Littlewood test with a slight extension. The method of proof used here is the same as in our note [2].

2. Let $f(x)$ be integrable and periodic, and

$$
f\left(x_{0}+t\right)+f\left(x_{0}-t\right)-2 f\left(x_{0}\right)=\phi\left(x_{0}, t\right)=\phi(t) \rightarrow 0, \quad t \rightarrow+0,
$$

then we have the following theorem:

TheOREM. The Fourier series $\subseteq[f]$ converges at the point $x_{0}$ if (2) is satisfied, and if for every $n$ there exists $m>n$ with $(m-n) \rightarrow \infty$, such that the following conditions are satisfied

$$
\sup _{\pi /(m+n) \leqq \theta \leqq 2 \pi /(m+n)} \sum_{k=1}^{\nu} \frac{1}{k+1}\left|\phi\left(2 \theta+\frac{2 k \pi}{m+n}\right)\right|=o(1)
$$

where $\nu=[(m+n) / 2(m-n)]$ and

$$
\sum_{k=n+1}^{m}\left(\left|a_{k}\right|+\left|b_{k}\right|\right)=o(1), \quad n \rightarrow \infty(m=m(n)) .
$$

Let $\left(\left|a_{n}\right|,\left|b_{n}\right|\right)=O\left(n^{-\delta}\right)$ and choose $m-n=\left[n^{\delta_{1}}\right]$ with $\delta_{1}<\delta$, then the left-hand side of (4) is less than $(m-n) \cdot O\left(n^{-\delta}\right)=O\left(n^{\delta_{1}-\delta}\right)=o(1)$.

Let (1) be satisfied, and so $\phi(t)=o(1 /|\log t|), t \rightarrow+0$; further let $(m-n)=\left[n^{\delta_{1}}\right]$, then the left-hand side of $(3)$ is less than

$$
\sup _{\pi /(m+n) \leqq \theta \leqq 2 \pi /(m+n)}\left|\phi\left(2 \theta+\frac{2 k_{1} \pi}{m+n}\right)\right| \sum_{k=1}^{\nu} \frac{1}{k+1},
$$

Received by the editors June 23, 1961 and, in revised form, September 18, 1961. 
for some $k_{1}$ satisfying $1 \leqq k_{1} \leqq[(m+n) / 2(m-n)]$. With the above $k_{1}$ we have

$$
2 \theta+\frac{2 k_{1} \pi}{m+n} \leqq O\left(\frac{1}{m-n}\right) \rightarrow 0, \quad m-n \rightarrow \infty,
$$

so that (5), in virtue of (1) does not exceed

$$
\frac{\epsilon_{k_{1}}}{\log (m-n)} \log (m+n)=O\left(\epsilon_{k_{1}}\right),
$$

where $1 \leqq k_{1} \leqq[(m+n) / 2(m-n)]$ and

$$
\max _{1 \leqq k_{1} \leqq\lfloor(m+n) / 2(m-n)\rfloor}\left|\epsilon_{k_{1}}\right| \rightarrow 0, \quad \text { as } n \rightarrow \infty .
$$

The above theorem therefore contains the Hardy-Littlewood test.

Proof of THEOREM. First, we suppose that the Fourier series $\mathfrak{S}[f]$ of $f(x)$ has an infinity of gaps $\left(n_{k}, n_{k+1}\right)$, for which $\left(n_{k+1}-n_{k}\right) \rightarrow \infty$. Let

$$
\Im[f]=\sum\left(a_{k} \cos n_{k} x+b_{k} \sin n_{k} x\right),
$$

take $n_{k+1}=m, n_{k}=n$, and denote by $D_{n}(t)$ and $K_{n}(t)$ the Dirichlet and Fejér kernels, i.e.,

$D_{n}(l)=\frac{1}{2}+\sum_{k=1}^{n} \cos k t, \quad(n+1) K_{n}(t)=\sum_{k=0}^{n} D_{k}(t)=\frac{\sin ^{2}(n+1) t / 2}{2 \sin ^{2} t / 2}$.

From the identity

$$
\begin{aligned}
m K_{m-1}(t)-n K_{n-1}(t) & \\
= & \sum_{k=n}^{m-1} D_{k}(t)=(m-n) D_{n}(t)+\sum_{k=1}^{m-n-1}(m-n-k) \cos (n+k) t
\end{aligned}
$$

we have, in virtue of lacunarity of $\subseteq[f]$ and (2)

$$
\begin{aligned}
s_{n}\left(x_{0}\right)-f\left(x_{0}\right)= & \frac{1}{\pi} \int_{0}^{\pi} \phi\left(x_{0}, t\right) D_{n}(t) d t \\
= & \frac{1}{\pi(m-n)}\left\{\int_{0}^{\pi / 2(m-n)}+\int_{\pi / 2(m-n)}^{\delta / 2}+\int_{8 / 2}^{\pi / 2}\right\} \\
& \cdot \phi\left(x_{0}, 2 t\right) \frac{\sin ^{2} m t-\sin ^{2} n t}{\sin ^{2} t} d t \\
= & I_{1}+I_{2}+I_{3}
\end{aligned}
$$

say. 
For $I_{1}$, we have

$$
I_{1}=\frac{1}{\pi} \int_{0}^{\pi / 2(m-n)} \frac{\phi(2 t)}{\sin t} \sin (m+n) t \frac{\sin (m-n) t}{(m-n) \sin t} d t .
$$

Since $\sin (m-n) t /(m-n) \sin t$ is non-negative and decreasing in $(0, \pi / 2(m-n))$ the second mean-value theorem, and the RiemannLebesgue theorem give

$$
I_{1}=\frac{1}{\pi} \int_{0}^{\xi} \frac{\phi(2 t)}{t} \sin (m+n) t d t+o(1), \quad 0 \leqq \xi \leqq \pi / 2(m-n) .
$$

The last integral can be written in the form

$$
I_{1}=\frac{1}{\pi} \sum_{k=1}^{\nu} \int_{(k-1) \pi /(m+n)}^{k \pi /(m+n)} \frac{\phi(2 t)}{t} \sin (m+n) t d t+o(1), \quad \nu \leqq\left[\frac{m+n}{2(m-n)}\right]
$$

and then can be majorized by

$$
\begin{aligned}
& \sup _{0 \leqq \lambda \leqq 2 \pi}\left|\phi\left(\frac{\lambda}{m+n}\right)\right| \\
& +\left|\int_{\pi /(m+n)}^{2 \pi /(m+n)}\left(\sum_{k=1}^{b} \frac{(-1)^{k} \phi(2 t+2 k \pi /(m+n))}{t+k \pi /(m+n)}\right) \sin (m+n) t d t\right| .
\end{aligned}
$$

By the first mean-value theorem the integral does not exceed

$$
\begin{aligned}
\frac{2}{m+n} \sup _{\pi /(m+n) \leqq \theta \leqq 2 \pi /(m+n)}\left|\sum_{k=1}^{\nu}(-1)^{k} \frac{\phi(2 \theta+2 k \pi /(m+n))}{\theta+k \pi /(m+n)}\right|, \\
\nu \leqq\left[\frac{m+n}{2(m-n)}\right]
\end{aligned}
$$

and consequently by (3) $I_{1}=o(1)$.

On the other hand, from (7) we obtain

$$
\left|I_{2}\right| \leqq \frac{1}{\pi(m-n)} \frac{\pi^{2}}{4} \frac{2(m-n)}{\pi} \sup _{\pi / 2(m-n)<\eta<\delta}\left|\phi\left(x_{0}, 2 \eta\right)\right|
$$

and

$$
\left|I_{3}\right| \leqq \frac{C}{(m-n) \delta^{2}} \quad(C=\text { const. }) .
$$

Now, we can choose for instance $\delta=(m-n)^{-1 / 4}$, so that by (2) $I_{2} \rightarrow 0$ and $I_{3} \rightarrow 0$ as $n \rightarrow \infty$. This proves that $s_{n}\left(x_{0}\right)-f\left(x_{0}\right) \rightarrow 0$ if $\mathfrak{S}[f]$ has gaps $\left(n_{k}, n_{k+1}\right)$ for which $\left(n_{k+1}-n_{k}\right) \rightarrow \infty$. 
In order to prove the theorem, it is enough, in view of (6) to prove that

$$
\left|\int_{0}^{\pi} \phi\left(x_{0}, t\right) \sum_{k=1}^{m-n-1}\left(1-\frac{k}{m-n}\right) \cos (n+k) t d t\right| \rightarrow 0,
$$

if $(m-n) \rightarrow \infty$, and if the condition (4) is satisfied. The last expression is equal to

$$
\begin{aligned}
&\left|\sum_{k=1}^{m-n-1}\left(1-\frac{k}{m-n}\right)\left(a_{n+k} \cos (n+k) x_{0}+b_{n+k} \sin (n+k) x_{0}\right)\right| \\
& \leqq \sum_{k=n+1}^{m}\left(\left|a_{k}\right|+\left|b_{k}\right|\right),
\end{aligned}
$$

which is equivalent to the condition (4) if $n \rightarrow \infty, m=m(n)$, so that (2), (3) and (4) imply $s_{n}\left(x_{0}\right)-f\left(x_{0}\right) \rightarrow 0$ as $n \rightarrow \infty$.

The author is indebted to the referee for many helpful comments.

\section{REFERENCES}

1. G. Sunouchi, Notes on Fourier analysis. I. On the convergence test of Fourier series, Math. Japon. 1 (1948), 41-44.

2. M. Tomic, A note on lacunary Fourier series, Proc. Amer. Math. Soc. 11 (1960), 460-462.

3. A. Zygmund, Trigonometric series, Vol. I, Cambridge Univ. Press, Cambridge, 1959.

Ecole des Mines, Belgrade, Yugoslavia 